\title{
Radar interferometry for SHM purposes
}

\author{
Milan Sokol ${ }^{1,}$, Katarína Lamperová ${ }^{1}$, Michal Venglár ${ }^{1}$, Monika Márföldi ${ }^{1}$, Lubomír \\ Prekop $^{1}$, Michal Krchňák ${ }^{2}$ \\ ${ }^{1}$ Slovak University of Technology in Bratislava, Faculty of Civil Engineering, Bratislava, Slovakia \\ ${ }^{2}$ ADC Projekt s.r.o., Bratislava, Slovakia
}

\begin{abstract}
The use of radar interferometry to measure dynamic response is now increasingly popular. The main advantage is a measurement of displacements. In this way, we obtain direct design parameters. Interferometric radar provides a cost-effective way to monitor bridges based on dynamic displacement measurement without traffic restrictions. The advantages and disadvantages are discussed. Comparing the numerical result with the test data provides a valuable opportunity to define a useful performance indicator.
\end{abstract}

\section{Introduction}

Structural health monitoring (SHM) of bridges is very important today [1, 2]. This is mainly due to the increasing age and in some cases of excessive load on the structure, which can cause considerable maintenance costs; in more serious cases, it is necessary to remove the damaged structure and replace it with a new one. The advantage of SHM is the early identification of failures that can be removed by minor repairs.

Measuring the dynamic response of bridges using conventional sensors (e.g. accelerometers) often requires partial or complete traffic restrictions on the bridge, which can be expensive. Radar interferometry is currently a widespread technology and many scientific teams consider this measurement method to be very advantageous [3 - 8]. One advantage is the direct measurement of structural displacements without the need for numerical integration, which is often the source of many errors. The measurement using IBIS-S radar is used to compare the phase shift of the electromagnetic wave, which was reflected from the measured object between two time points [5,9]. Another advantage of this technology is that the measurement of the dynamic response of the structure is performed from a location relatively distant from the structure itself. For this reason, there is no need to restrict traffic on the bridge.

Despite these advantages, there have also been errors in measuring bridges and large structures. The first disadvantage is the result of imaging radar capability only in 1D space. There may be an error in measuring displacements of different points that are the same distance from the radar. The second disadvantage is that the radar provides a measurement of the displacement of the observed point along the radar sensor field of view (radial displacement). For this reason, it is necessary to evaluate the real displacement, which must

\footnotetext{
* Corresponding author: milan.sokol@stuba.sk
} 
already be based on previous knowledge of the direction of motion of the point of interest, so it is necessary to perform the initial measurements.

\section{Measurement of Dynamic Response of Various Types of Bridge Structures}

The speed and accuracy of interferometric radar measurements largely depend on the skill and experience of the person operating the equipment. It is important to find a suitable place for the location of the radar. A suitable location should allow for sufficient stabilization of the radar and must be large enough to handle the device. One of the disadvantages is the very high sensitivity of the device to environmental impacts (grass, bushes, branches, etc.). However, this can often be eliminated by removing interfering objects from the radar measurement range before starting the measurement. The accuracy of the measurement depends on the ratio of signal intensity to the noise of the measured point and thus depends on the distance of this point. The further the measured point is, the lower the intensity of the reflected signal is. At longer distances, the reflectance of the target can be increased by reflectors. Depending on the intensity of the reflected signal, a measurement accuracy of 0.1 $\mathrm{mm}$ can be achieved [9].

It is very important to precisely target the device with respect to the measured structure. Interferometric radar measures changes in radial distances of points. For bridge structures, the vertical distance of the device from the measured point is very important for the conversion of radial displacement in the vertical direction. Inaccurate determination of the distance can be a source of errors in the conversion.

We were able to perform dynamic measurements of displacements of various types of bridge structures without limitation and with partial limitation of traffic on the bridge. Otherwise, concrete and steel bridge structures must be measured.

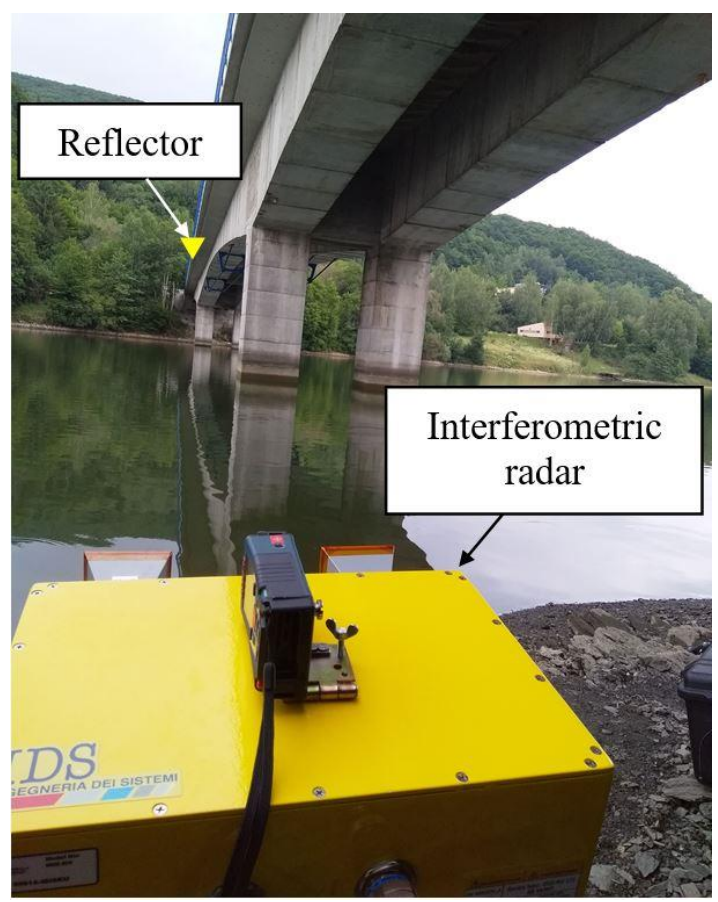

Fig. 1. Measured structure - concrete road bridge. 


\subsection{Measurement of Displacements of Concrete Bridge}

One of the measured structures was the bridge over the Ružín water reservoir (three-span concrete road bridge) (Fig. 1.). On this structure, there are hardly any points that would naturally reflect signals back to the device. When measuring this type of structure, it is usually necessary to place reflectors in places where we want to measure dynamic displacements. The measured displacements were then compared to numerical calculations.

This dynamic load test was carried out with a partial restriction in traffic on the bridge. There was a two-axle load vehicle that passed at various speeds through an artificial obstacle located in the middle of the span of the structure. A reflector was placed at this point. In addition to the interferometric radar, the dynamic response of the structure was also recorded using accelerometers and strain gauges. At the same time, the test was also recorded with a video camera, which allowed to verify the vehicle speed and its correct passage through the obstacle.

\subsection{Measurement of Displacements of Steel Bridge}

Another measured structure was the bridge over the Torysa River (three-span steel railway bridge) (Fig. 2.). This measurement was made without traffic restrictions on the bridge. This test was also recorded with a video camera. Passing train data, such as locomotive type and wagon type, were determined according to the timetable. For the purpose of numerical calculations and subsequent comparison with the measured displacements, specific technical information such as the number of axles, axle spacing, and weight was identified.

No reflectors were needed to measure the displacement of this structure. The signal was naturally reflected from the rectangular connections of the structural members.

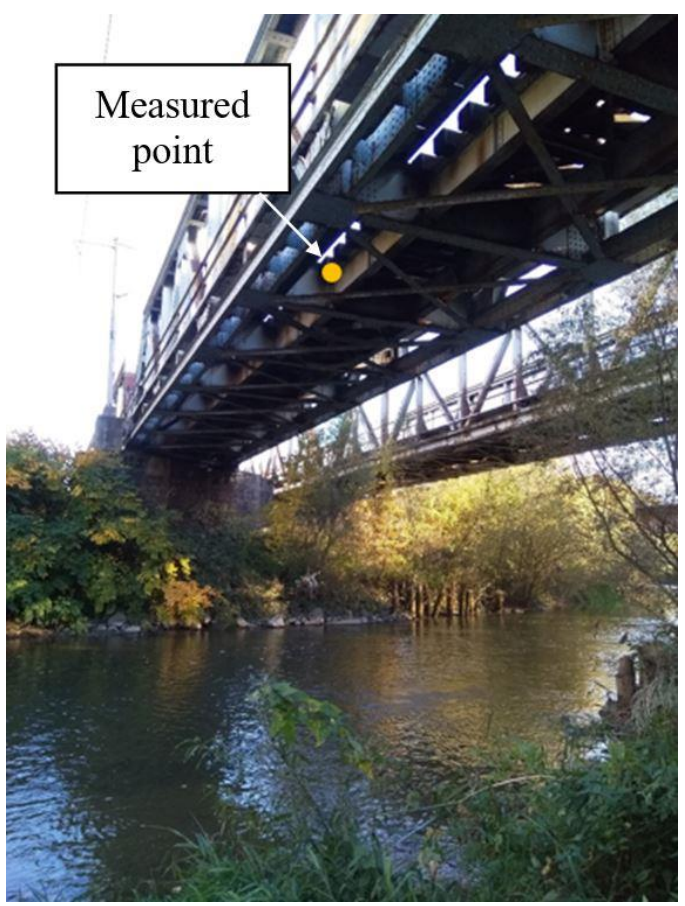

Fig. 2. Measured structure - steel railway bridge. 


\section{Evaluation of experimental results and comparison with calculation}

Some of the design parameters, such as dynamic coefficients and natural frequencies, can be obtained directly from the measured dynamic displacements of the structure. If we know the traffic situation on the bridge during measurement, it is possible to do numerical simulations. In this way, numerical models can be verified and calibrated. Comparing the numerical results with the test data defines a useful performance indicator (PI). This indicator shows how the result of the numerical model corresponds to the test data. A validated and calibrated numerical model may be a suitable basis for structural health monitoring (SHM).

From the displacement records, some natural frequencies at the vertical oscillation of the unloaded structure and the loaded structure were determined. The logarithmic decrement of damping was determined from the same records. These values were compared to numerical calculations and measurement results using accelerometers and strain gauges.

\subsection{Dynamic amplification factor}

From the displacement records, the dynamic amplification factor was determined according to equation (1).

$$
\delta_{o b s}=\frac{d_{\max }}{d_{m}}
$$

In this equation, $d_{\max }$ is the maximum value of the measured deflection at the measured point when passing the test vehicle, and $d_{m}$ is the maximum value of the measured displacement from the static action of the vehicle. The measured values were assessed according to the criteria in [10]. The critical speed was determined according to the dynamic coefficient dependence on the speed of passing test vehicle.

\subsection{Performance indicator}

Performance indicator was determined according to equation (2).

$$
P I=\frac{d_{\text {num }}}{d_{\text {test }}}
$$

In this equation, $d_{\text {num }}$ is the maximum value of the calculated displacement of the assessed point from the static load, and $d_{\text {test }}$ is the maximum value of the measured displacement of the assessed point from the static load.

\section{Conclusions}

Radar interferometry measurements have several advantages over conventional sensors. One of them is the need to place the sensors in places representing the structure. With poor availability of these locations, this can be problematic with conventional sensors. Other disadvantages include the need to use a large number of cables.

With proper placement and adjustment of interferometric radar, the measurement is fast and relatively accurate. In this way, we obtain direct design parameters, such as dynamic coefficients and natural frequencies. This technology provides a cost-effective way to monitor bridges based on dynamic displacement measurement without traffic restrictions. 
Comparing the numerical result with the test data provides a valuable opportunity to define a useful performance indicator.

This paper has been supported by the grant No. 1/0749/19 provided by VEGA Agency of Ministry of Education, Science, Research and Sport of the Slovak republic. It was also supported by grant from research program of Slovak University of Technology -Young researchers 2019.

\section{References}

1. Chowdhury, F.H., Raihan, M.T. and Islam, G.M.S. IABSE-JSCE Joint Conference on Advances in Bridge Engineering-III. (Dhaka, Bangladesh, 2015).

2. Farrar, C. R., Lynch, J. P., Michaels, J. E. Proceedings of the IEEE. (2016) Vol. 104 (8).

3. Kuras, P. et al. Bridge Maintenance, Safety, Management, Resilience and Sustainability. (Taylor \& Francis Group, London, UK, 2012).

4. Gentile, C.: Radar Technology. (InTech, Rijeka, Croatia, 2010).

5. Gocal, J. et al. Determination of displacements and vibrations of engineering structures using ground-based radar interferometry. (Wydawnictwa AGH, Krakow, 2013).

6. Raventós, J., Couso, C., García, J. INGEO 2017 Proceedings of the 7th International Conference on Engineering Surveying. (Lisabon, Portugal, 2017).

7. Li, H.-N. et al. Structural Monitoring and Maintenance. (Techno-Press, 2016).

8. Sokol, M., Venglár, M., Ároch, R., Kopáčik, A.; Kyrinovič, P.; Erdélyi, J., Šišmišová, Z., Lamperová, K. 39th IABSE Symposium - Engineering the Future. (Vancouver, Canada, 2017).

9. Ingegneria Dei Sistemi (IDS) S.p.A.: IBIS-S System ver. 1.0 - User Manual. (Pisa, 2010).

10. STN 73 6209: Load testing of bridges, SÚTN Bratislava, 09, (1987) 